\title{
Exploring the cultural flexibility of the ACT model as an effective therapeutic group intervention for Turkish speaking communities in East London
}

\author{
Aradhana Perry ${ }^{1}$, Chelsea Gardener ${ }^{2}$, Joseph E. Oliver ${ }^{3}$, Çiğdem Taş ${ }^{4}$ and \\ Cansu Özenç ${ }^{5}$
}

${ }^{1}$ City and Hackney BME Access Service, East London NHS Foundation Trust, City and Hackney Directorate, Department of Psychology

${ }^{2}$ STEP Team, South London and Maudsley NHS Foundation Trust

${ }^{3}$ University College London

${ }^{4}$ Mental Health Co-Ordinator, Derman

${ }^{5}$ John Howard Centre, East London NHS Foundation Trust

Received 31 May 2017; Accepted 12 March 2018

\begin{abstract}
This study describes a successful community-based partnership project between statutory and third-sector services in East London; The City and Hackney Black and Minority Ethnic (BME) Access Service [East London NHS Foundation Trust (ELFT)] collaborated with Derman, a local community organization supporting the well-being of Turkish-speaking communities, to explore the cultural adaptability of an empirically supported, third-wave cognitive behavioural intervention, Acceptance and Commitment Therapy (ACT). The aim was to develop a culturally acceptable group that was responsive to the therapeutic needs of participants from Turkish-speaking communities. The study implemented a mixed-method analysis with a one group pre/post-test design to examine the effectiveness of a 7-session culturally adapted ACT group intervention and a descriptive approach was implemented to assess usefulness, relevance and acceptability. Results demonstrated an overall positive effect of the culturally adapted ACT intervention in terms of both symptoms and patient-reported outcomes. Participants showed significant improvements on measures of depression $(p=0.014)$, anxiety $(p=0.041)$ and psychological distress $(p=0.003)$. The magnitude of these changes was categorized as large, with effect sizes from 0.90 to 2.03. Qualitative responses indicated that the group was experienced as enjoyable and useful and was considered to be an accessible and acceptable therapeutic format. Although a pilot within clinical practice, the findings provide preliminary support for the clinical utility of ACT as an effective, culturally acceptable therapeutic approach for Turkish-speaking communities living in an urban UK setting. The study highlights the importance of culturally appropriate service development and a need for further research within this area.
\end{abstract}

Key words: ACT, Acceptance and Commitment Therapy, cultural flexibility, cultural adaptation, BME, Turkish speaking communities 


\section{Introduction}

Acceptance and Commitment Therapy (ACT) is a trans-diagnostic, contextual cognitive behavioural therapy that seeks to improve well-being through processes that promote steps toward valued actions, whilst reducing the impact of unhelpful psychological processes of cognitive fusion and emotional and behavioural avoidance (Hayes et al., 2006). The overall aim is to increase psychological flexibility in the face of unwanted distressing thoughts and emotions (Hayes et al., 2012). There is now a significant body of treatment outcome research suggesting that the integration of acceptance, mindfulness and compassion-focused principles within cognitive behavioural therapies is effective in improving well-being and quality of life (A-Tjak et al., 2015; Ruiz, 2010). Despite substantial literature for the efficacy of these interventions across a range of disorders, there is only a limited evidence base into the generalizability of these findings and applicability to different BME populations in a UK context.

Cultural and socio-demographic factors influence the way people experience distress, symptom expression and help seeking behaviours (Bhui et al., 2015; Furnham and Malik, 1994; Rathod et al., 2013). Cultural modifications are therefore necessary and important considerations when attempting to demonstrate the clinical and cultural competency of a treatment approach for diverse communities in terms of inclusion of cultural knowledge, therapeutic process and equitable service delivery (Tseng, 2004).

Cultural adaptation is the 'systematic modification of an evidence-based treatment or intervention protocol to consider language, culture and context in such a way that it is compatible with the client's cultural patterns, meanings and values' (Bernal et al., 2009, p. 362). A critical aspect of culturally adapting an intervention to the needs of a particular community is the integration of both 'top-down' and 'bottom-up' approaches. The Ecological Validity Model (EVM) proposed by Bernal et al. (1995) is based on a bottom-up communityengagement approach and advocates building collaborative relationships with community stakeholders in order to increase the social validity (i.e. acceptability) of the adapted intervention. They suggest that culturally adapted interventions are more effective when there is congruence between the cultural perspective of the community and the properties of a particular therapeutic intervention, with poor response to treatment possibly indicative of poor social validity (Bernal et al., 1995; Bernal et al., 2009; La Roche and Lustig, 2013).

Empirical support for this proposition, derives from a meta-analysis of 76 studies, which found a weighted average effect size of $d=.45$, indicating a moderately strong benefit of culturally adapted interventions in client improvements across a variety of conditions and outcome measures (Griner and Smith, 2006). Literature exploring aspects of adaptations indicate that factors such as ethnic matching, provision of interventions in the client's native language and adaptations tailored to a specific cultural context are more efficacious, with an increased likelihood of enhanced engagement, retention and satisfaction (Griner and Smith, 2006). Delivery within a local community setting enhances accessibility and consultation with individuals familiar with the clients' culture and being inclusive of support resources that are available within collectivist cultural contexts (e.g. spiritual traditions, extended family) also facilitated positive outcomes (Griner and Smith, 2006). In addition, a recent systematic review on therapeutic interventions and communications with BME communities concluded that the cultural adaptation of western psychological therapy models improved therapeutic outcomes when there was an emphasis on empowerment (Bhui et al., 2015). 
Currently, within the ACT literature there are several types of culturally adapted ACT interventions and successful evaluations facilitating positive outcomes across diverse, nonwestern populations, such as in Iran, India and South Africa (e.g. Hoseini et al., 2014; Fuchs et al., 2013; Woidneck et al., 2012; Hall et al., 2011; Lundgren et al., 2006, 2008). Other research has also found that ACT training workshops in Sierra Leone were both culturally applicable and accessible (White and Ebert, 2014; Stewart et al., 2016). This growing body of literature explores the efficacy of adapting ACT for non-western collectivist cultures and coupled with the wider literature on cultural adaptations, highlights the need to explicitly incorporate cultural content, context and values relevant to the client's well-being into the intervention (Bernal et al., 2009; Griner and Smith, 2006).

\section{Cultural adaptation of ACT to a Turkish-speaking context}

The Turkish diaspora in Europe consists of three main groups: Kurdish, mainland Turks and Turkish Cypriot communities (Eylem et al., 2016). These groups migrated to Europe for different historical and political reasons (Enneli et al., 2005). Besides the cultural, ethnic and religious differences between these groups, they share commonalities such as language and collectivist values (e.g. duty, roles, relatedness, interdependence, familism, community) based on their history of interaction (Eylem et al., 2016; Kuzulugil, 2010). Experiences and expressions of distress are thus formulated in terms of a shared knowledge and shaped by cultural, sociopolitical and historical contextual factors (Eylem et al., 2016).

ACT is a highly pragmatic, experiential and collaborative therapeutic approach based on connecting people to important values and using this information to guide therapeutic process and promote flexible and purposeful living (Masuda, 2014). The emphasis on values and context are two central tenets of the ACT model that offer potential for cultural adaptability for Turkish-speaking communities given their collectivist structure and many experiences of distress being located in contextual circumstances (Fuchs et al., 2013; Woidneck et al., 2012) such as familial relationships (e.g. marital and/or inter-generational conflict) and collective experiences of loss, displacement, being a refugee, war and genocide. This is congruent with the philosophical orientation and contextual therapeutic stance of ACT (Masuda, 2014; Hayes et al., 2006), which then teaches mindfulness and acceptance processes and skills (such as present moment awareness and defusion) to change an individual's relationship to unwanted internal experiences (painful thoughts, feelings, memories and sensations) rather than changing the experience itself. Furthermore, ACT uses the behavioural change process of 'committed action' (in the service of values) to open up choices that may help to overcome hopelessness and foster a greater sense of empowerment, meaning and purpose. Thus, the clinical utility of ACT for Turkish speaking clients may be in helping to enhance their sense of personal agency and emotional resilience through developing psychological flexibility in response to distress so people learn how to live well in the presence of difficult life circumstances.

\section{Service context}

The 'Psychological Therapies Alliance' (PTA) was implemented by the City and Hackney Clinical Commissioning Group to integrate care pathways between statutory and third-sector providers of psychological and psychosocial interventions and increase equity of access for 
BME communities (City and Hackney Clinical Commissioning Group, 2015). Developing specific provision involving collaboration with targeted communities has been highlighted in the literature relating to the importance of cultural adaptations outlined above (Bhui and Olajide, 1999; Bhui et al., 2000, 2015). This recognizes the important role of thirdsector partnerships in the development and delivery of therapeutic services, with subsequent implications for clinical practice in terms of accessibility and utilization of talking therapy services for BME communities.

As a highly trusted third sector organization, Derman provides a range of health-related services to Kurdish, Turkish, Turkish Cypriot and Eastern European Turkish-speaking people (mainly asylum seekers and people from refugee backgrounds) in Hackney and plays a central role as a bridge between community members and statutory services. They have invaluable knowledge of the physical and mental health needs of the local communities they serve and are often the first line of assistance to those seeking help.

The City and Hackney BME Access Service (ELFT) is located within secondary care and works in close partnership with key third-sector organizations and internal stakeholders to address barriers to accessing appropriate talking therapies among local BME communities. The service is based on the Centre for Ethnicity and Health's Community Engagement Model (Fountain et al., 2007) and employs a community psychology approach that aims to meet the mental health needs of under-represented groups through equitable access, improved service experience and outcomes.

The PTA commissioned this partnership initiative in line with national government directives on BME positive practice guidance (promoting joint working between statutory services, local third-sector organizations and faith groups; Department of Health, 2009) and in relation to previous research forming part of the national community engagement NIMHE Mental Health Programme ${ }^{1}$ which recommended improving the provision and quality of culturally appropriate talking therapies for Turkish-speaking communities (Tas et al., 2008). A project between the City and Hackney BME Access Service and Derman was therefore established to pilot the development and delivery of a culturally adapted ACT group intervention, with the over-arching purpose of improving the acceptability and accessibility of talking therapies within these communities.

\section{Aims of the ACT group}

(1) To explore the cultural flexibility of the ACT model and produce a culturally relevant adaptation of an existing ACT group therapy protocol ('ACT - Promoting Mental Health and Resilience'; Livheim, 2014) that is responsive to the therapeutic needs of participants from Turkish-speaking communities.

(2) To assess the acceptability of the culturally adapted version of the ACT group format for members of the Turkish-speaking communities.

(3) To measure if the culturally adapted ACT group intervention improved psychological well-being amongst Turkish-speaking participants.

\footnotetext{
${ }^{1}$ Funded by the National Institute for Mental Health in England and supported by the Centre for Ethnicity and Health, University of Central Lancashire.
} 


\section{Method}

\section{Cultural adaptation framework}

The ecological validity model (Bernal et al., 1995) was chosen to guide the process of modifying an existing ACT treatment protocol as this framework allowed the core principles of ACT to be retained but with adequate flexibility for cultural adaptation, thereby preserving validity to the original treatment (Rathod et al., 2013). The ecological validity model delineates eight dimensions to consider when culturally adapting an intervention. These include the use of appropriate language, persons (cultural similarities/differences between the client and clinician which shape the therapeutic relationship), metaphors (symbols and concepts), content (cultural knowledge), concepts (treatment concepts that are culturally congruent), goals (that support adaptive cultural values), methods (cultural enhancement of treatment methods) and context (consideration of acculturation, social context).

\section{Consultation}

The therapeutic protocol described here is based on the 7-session 'ACT - Promoting Mental Health and Resilience' group therapy framework developed by Livheim (2014) and adapted by the City and Hackney BME Access Service in collaboration with Derman. The main structure of the original Livheim ACT group format was maintained but different elements were distilled for cultural appropriateness and accessibility.

The process of adaptation was informed by two 3-hour community consultations with Derman. Nine participants attended the first session and seven attended the second. Participants included the chief executive officer of Derman, mental health advocates, counsellors, support workers and administrative staff. Consultations allowed space for a detailed discussion of the main components of the Livheim ACT group programme to be carefully examined for cultural relevance and produced a number of important suggestions incorporating cultural understandings of mental health and well-being within a Turkish-speaking cultural context.

Turkish-speaking cultures are rich in proverbs, poems, songs and stories and so efforts were made to link cultural knowledge to key ACT concepts and practices by using cultural bridging techniques such as culturally relevant metaphors, values, traditions, common folk stories (e.g. the 'farmer and the horse'), cultural imagery and inspirational role models.

For example, images of the revered Sufi poet and philosopher Mevlânâ (Rūmī) were used to represent the embodiment of openness, kindness and compassion to life's experiences, self and others. Popular teachings and poems (e.g. 'The Guest House'; 'Come, Come, Whoever You Are') were used to illustrate core ACT processes and mindfulness skills (contact with the present moment, acceptance, self as context, defusion) and cultivate a welcoming stance toward all internal experiences (Mirdal, 2012).

Culturally familiar, non-technical language and video material from popular Turkish media were also used to explain therapeutic concepts. For example, a short film showing a successful Turkish businessman living with blindness from birth was used to inspire perseverance and persisting in the service of chosen values.

A culturally syntonic translation of the material was made and the completed 7-session adapted group therapy format was written up in both English and Turkish. The final group protocol combined didactic elements, group discussion, role plays and experiential group exercises (e.g. mindfulness [mindful eating with Turkish delight], life compass, 'passengers 

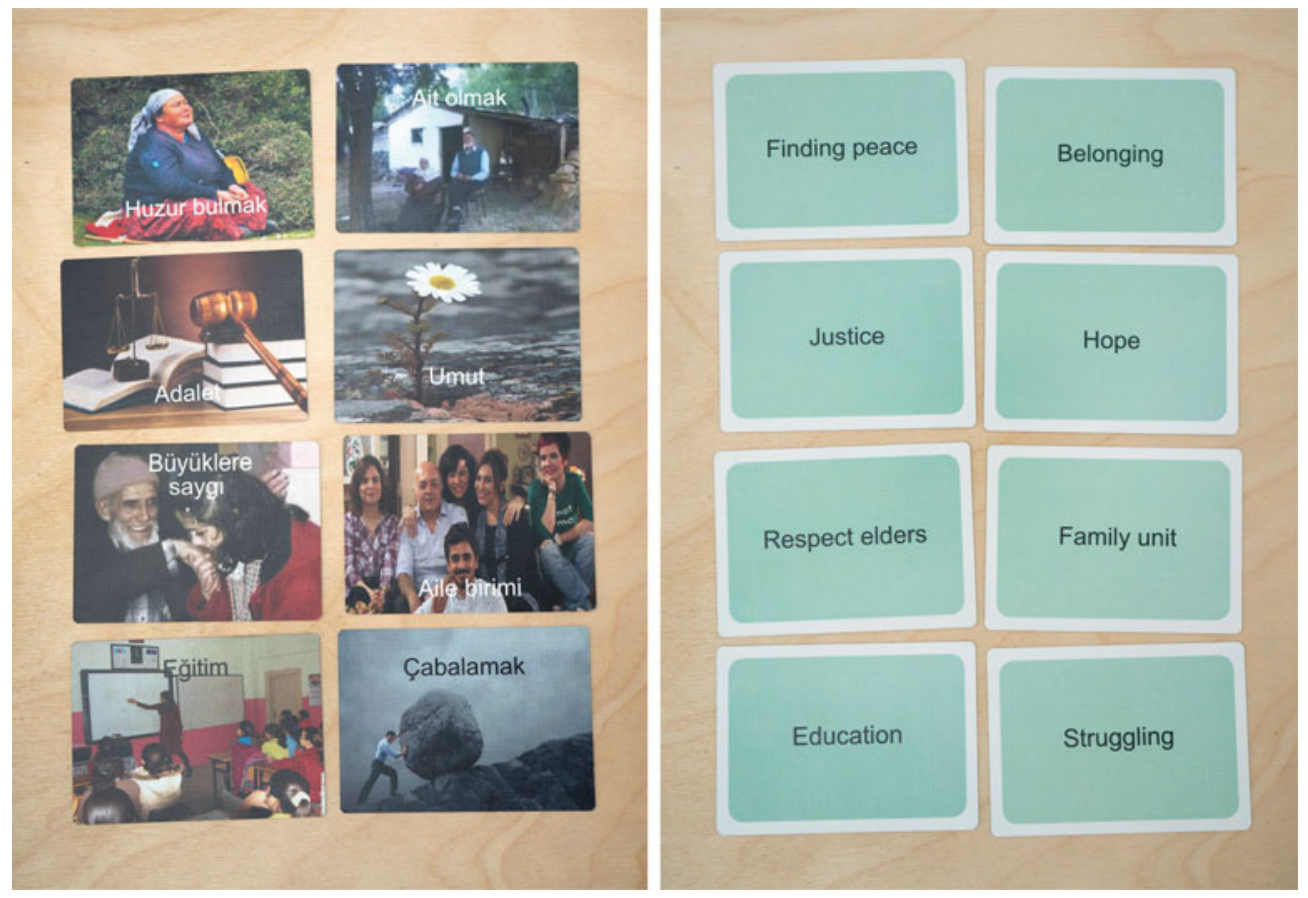

Figure 1. Turkish collectivist 'value cards'

on the bus', Chinese finger traps). The culturally adapted ACT group protocol was renamed: 'Farkındalık ve Şevkatle Kabullenme' ('Acceptance with Mindfulness and Compassion').

The adaptation process also produced shared resources so that the group could be delivered in both statutory and voluntary sectors in the future to support sustainability. Resources included collectivist value cards specifically developed for Turkish-speaking contexts. For example, the cultural value 'Çabalamak' ('Struggling') is imbued with the qualities of courage, perseverance and strength to overcome difficulty. See Fig. 1 for details. Two audio CDs containing acceptance, mindfulness and compassion focused exercises recorded in Turkish were also provided to support home practice.

\section{Design}

The study implemented a mixed-method analysis with a one group pre/post-test design to examine the effectiveness of the culturally adapted ACT group and a descriptive approach was implemented to assess the usefulness, relevance and acceptability of the group from qualitative feedback.

\section{Procedure}

The ACT group consisted of 2-hour weekly sessions over 8 weeks. The group was cofacilitated by the (English speaking) BME Access Service clinical psychologist and bilingual 
mental health coordinator from Derman. The group was predominantly delivered in the Turkish language and a bilingual advocate provided additional language support within the group by translating group material from English to Turkish and Turkish to English for the clinical psychologist. The final session was used for completing post-intervention measures and provided an opportunity to collate service user satisfaction feedback. The ending of the group was celebrated with food and participants were awarded with a certificate for completing the group and given copies of the CDs with acceptance and compassion exercises.

\section{Sampling and recruitment}

Referrals were received via the City and Hackney mental health network (City and Hackney MIND), from GPs, other local authorities or self-referral, and group participants were randomly selected from Derman's mental health waiting list. Clients identified as appropriate were offered the option of attending the ACT group. Those who agreed were jointly assessed for suitability by the BME Access Service clinical psychologist and mental health coordinator from Derman.

\section{Group inclusion criteria}

The target group population were Turkish-speaking men and women representing the Turkish diaspora (Kurdish, Turkish and Turkish Cypriot) who access Derman services, aged between 18 and 65 years. Participants needed to be motivated to attend group therapy for issues related to stress, anxiety and low mood.

Clients were excluded from the group if they:

- Were experiencing acute symptoms of psychosis;

- Had a diagnosed learning disability;

- Presented with ambivalence or low motivation to engage in group work;

- Historically had a high 'did not attend' rate;

- Presented with a primary problem of gambling, drugs, alcohol;

- Were in early stages of bereavement and presenting with acute grief.

\section{Setting}

The ACT group was delivered in a spacious group meeting room within the community setting of Derman, a venue that was considered to be a safe and accessible space for community members.

\section{Group participants}

The group was closed and a total of eight participants were recruited. The main presenting difficulties of participants were: relationship problems (marital, intergenerational), chronic pain, anxiety, depression, low self-esteem and long-standing health problems. 


\section{Measures}

Three pre- and post-test measures were used to evaluate psychological well-being, which were standardized measures across the service context. All measures were translated into Turkish and verbally administered:

- A shortened version of the CORE (CORE-OM; Barkham et al., 2013) measuring levels of psychological distress.

- The depression module of the Patient Health Questionnaire (PHQ-9; Kroenke and Spitzer, 2002), which measured depression symptoms and severity.

- The GAD-7 (Spitzer et al., 2006) measuring common anxiety symptoms and severity.

Two patient-rated experience measures were used to capture the views and experiences of participants: the Most Important Event (MIE) questions were asked after each session: 'What was the most important/helpful thing that happened here today?' and 'What was the least important/unhelpful thing that happened here today?'. Responses were recorded verbatim, providing qualitative feedback from each session. These questions were chosen as they linked to previous literature on methods for identifying what is helpful for clients and assessing therapeutic factors in group therapy associated with client outcomes (Berzon et al., 1963; Bloch et al., 1979; Lieberman et al., 1973; Yalom, 1975).

Secondly, a brief service user satisfaction questionnaire was developed and administered in the format of an evaluation interview at the end of the group intervention (session 8). Participants were also offered a reflective space in this session to review the group as a whole and provide individual feedback.

\section{Analysis}

Data were analysed using SPSS version 22.

\section{Quantitative analysis}

Paired $t$-tests were performed to compare pre- and post-test measures, as data met assumptions of normality. Effect sizes were then calculated, as outlined by Lakens (2013). As Cohen's $d$ has been shown to give a slight bias in effect size within small sample sizes (Borenstein et al., 2009; Fuchs et al., 2013; Lakens, 2013), a more conservative measure of effect size was calculated using Hedges' gav or grm (as recommended by Lakens, 2013). This Hedges correction can be interpreted with Cohen's convention of effects sizes.

Given the small sample size, individual level change was assessed with reliable change indices (RCI), where change is reliable when greater than might be expected by chance, given the reliability of the measure. Where reliable change was detected, further analyses were performed to determine what percentage of reliably improved scores could also be considered clinically significant. Criterion C of Jacobson and Truax (1991) was used to define clinically significant change (CSC) as a person's score being in the 'clinical' range pre-treatment and below the clinical cut-off post-treatment.

The quantitative data from the service user satisfaction survey were collated to present frequency feedback. 
Table 1. Pre-and post-intervention scores on all outcome measures and associated test of difference

\begin{tabular}{llllll}
\hline Variable & Mean T1 $(S D)$ & Mean T2 $(S D)$ & $t$ (d.f.) & $p$ value & $\begin{array}{l}\text { Effect } \\
\text { size }\left(g_{\text {rm }}\right)\end{array}$ \\
\hline Psychological distress $(n=7)$ & $24.57(6.37)$ & $11.43(5.74)$ & $4.944(6)$ & $0.003^{* *}$ & 2.03 \\
Depression $(n=7)$ & $17.36(8.05)$ & $10.57(6.11)$ & $3.445(6)$ & $0.014^{*}$ & $0.90^{\ddagger}$ \\
Anxiety $(n=7)$ & $15.86(6.64)$ & $9(4.97)$ & $2.588(6)$ & $0.041^{*}$ & 1.09 \\
\hline
\end{tabular}

${ }^{*}$ Significant at $p \leq 0.05 ;{ }^{* *}$ significant at $p \leq 0.01 ;{ }^{\dagger}$ indicates effect size $g_{\text {av }}$.

\section{Qualitative analysis}

A thematic analysis was conducted on the data derived from the MIE questions every session, the qualitative responses on the service user satisfaction survey and the verbatim recording of the reflective group discussion post-intervention. The thematic analysis followed steps outlined in Braun and Clarke (2006), which included six steps: 'familiarization, searching for themes, review of themes, definition and naming, and production of the report'. Emergent themes were discussed with another independent rater to check that they were grounded in the data and reduce the risk of idiosyncratic readings of the data being sustained.

\section{Results}

One participant dropped out due to obtaining paid employment; therefore their data was omitted and the results section describes data for the remaining seven participants.

\section{Group attendance}

The group was well attended, with attendance above $70 \%$ for each session.

\section{Demographics}

All participants were first generation (i.e. born outside the UK), their ages ranged from 42 to 62 years (average age approximately 55 years) and the majority of participants were female, married and from Turkish and Turkish Cypriot ethnic backgrounds. The average length of time participants were known to Derman was approximately 4.5 years and the majority of the participants had used the counselling and group activity services at Derman.

\section{Treatment outcomes}

The results indicate a statistically significant improvement in psychological distress $(p=0.003)$, depression $(p=0.014)$ and anxiety $(p=0.041)$ scores following the group (see Table 1). The mean decrease for depression scores was 6.79 (5.21) with a 95\% confidence interval ranging from 1.97 to 11.61 . The mean decrease for psychological distress scores was 13.14 (7.03) with a $95 \%$ confidence interval ranging from 6.64 to 19.65 . The mean decrease for anxiety scores was 6.86 (7.01) with a $95 \%$ confidence interval ranging from 0.37 to 13.34 . 
Table 2. Reliable change for outcome measures by severity; and reliable and clinical significant change on outcome measures

\begin{tabular}{lccc}
\hline Baseline scores & Deterioration & No change & Improvement \\
\hline CORE-10 & & & \\
Below cut-off $(<11)$ & 0 & 0 & 0 \\
Above cut-off & 0 & 1 & 6 \\
Total $(n=7)$ & $0(0 \%)$ & $1(14.3 \%)$ & $6(85.7 \%)$ \\
Reliable and clinically significant improvement & - & - & $3(42.8 \%)$ \\
PHQ-9 & 0 & 2 & 0 \\
Below cut-off $(<10)$ & 0 & 0 & 5 \\
Above cut-off & $0(0 \%)$ & $2(28.6 \%)$ & $5(71.4 \%)$ \\
Total $(n=7)$ & - & - & $2(28.6 \%)$ \\
Reliable and clinically significant improvement & - & 2 & 0 \\
GAD-7 & 0 & 0 & 5 \\
Below cut-off $(<10)$ & 0 & $2(28.6 \%)$ & $5(71.4 \%)$ \\
Above cut-off & $0 \%)$ & - & $2(28.6 \%)$ \\
Total $(n=7)$ & - & & \\
Reliable and clinically significant improvement & - & &
\end{tabular}

The magnitude of these changes were categorized as large, with effect sizes ranging from 0.90 to 2.03 (see Table 1).

\section{Individual level analyses of reliable change indices and clinically significant change}

Table 2 shows the percentage of participants whose changes in scores can be considered reliable. For the CORE-10, all seven participants scored above clinical cut-off at baseline, and $85.7 \%$ showed a reliable improvement on this measure of psychological distress postintervention. For both the PHQ-9 and GAD-7 measures, all five participants who scored above the clinical cut-off at baseline indicated a reliable improvement in both depression and anxiety post-intervention $(71.4 \%)$. There was no reliable deterioration on any of the measures.

Further analyses were carried out to determine what percentage of reliably improved scores could also be considered as clinically significant changes (CSC). For the CORE-10, 42.8\% $(n=3)$ of participants showed both reliable and clinically significant improvement, meaning that following the group they fell below the clinical cut-off for experiencing psychological distress (see Table 2). For the other three participants whose scores reliably improved on the CORE-10 but did not meet criteria for CSC (did not fall below clinical cut-off range), their scores moved from the severe range to either the moderate $(n=2)$ or the moderate-severe range $(n=1)$.

Table 2 shows that $28.6 \%(n=2)$ of participants showed both reliable and clinically significant improvements on the PHQ-9 and the GAD-7, meaning that post-intervention their scores fell below the clinical cut-off for depression and anxiety. For the other three participants whose scores reliably improved on the PHQ-9 but did not meet criteria for CSC (did not fall below clinical cut-off range), two participants' scores moved from the severe range to either the moderate $(n=1)$ or the moderate-severe range $(n=1)$, and one participant's scores reduced within the severe range for depression. For the GAD-7, of the three participants whose 


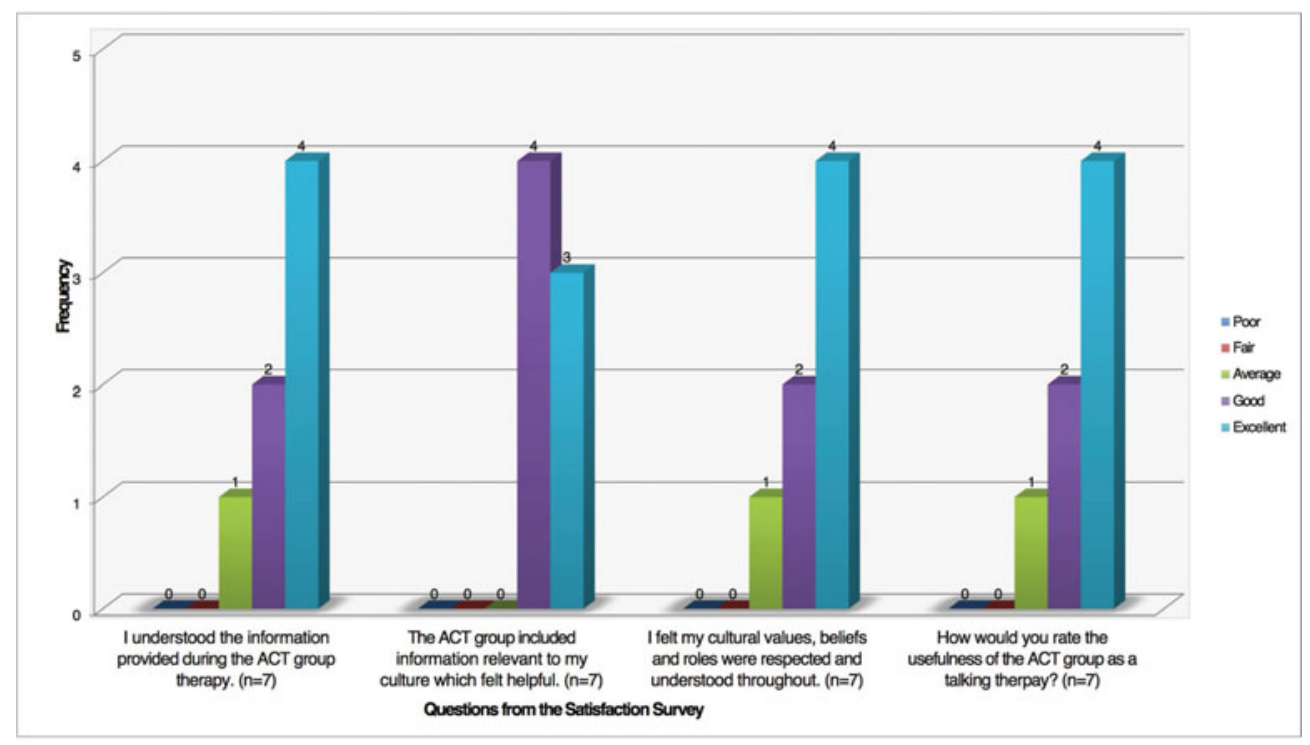

Figure 2. Frequency of responses to questions from the Service User Satisfaction Survey

scores reliably improved but did not meet criteria for CSC (did not fall below clinical cut-off range), two participants' scores moved from the severe range to moderate $(n=2)$ and one participant's scores reduced within the severe range for anxiety.

\section{Descriptive information from the service user satisfaction survey}

All seven participants reported finding it helpful to talk about their problems and most participants said they understood the information provided in the group. The majority of participants thought that the ACT group was 'good' or 'excellent' at including relevant cultural information in a helpful way and agreed that aspects of their culture (roles, values and beliefs) were respected and understood. Similarly, the group was rated highly for usefulness as a talking therapy by the majority of participants, except one who rated it as average usefulness. See Fig. 2 for details.

\section{Thematic analysis}

The thematic analysis identified three over-arching meta-themes (with relating sub-themes) which were:

- Group Process (sub-themes: Interpersonal factors; A safe space)

- Change Factors (sub-themes: Emotional transitions; New perspectives; Different actions)

- Reflections/Considerations (sub-themes: Group feedback; Interpreting language; Use of metaphor)

The text identifies quotes that encapsulate the themes and Table 3 presents additional indicative quotes of the themes identified. 
Table 3. Themes and indicative quotes

\begin{tabular}{|c|c|c|}
\hline Master theme & Sub-themes & Some indicative quotes from the data \\
\hline $\begin{array}{l}\text { 1. Group } \\
\text { Process }\end{array}$ & $\begin{array}{l}\text { Interpersonal } \\
\text { factors }\end{array}$ & $\begin{array}{l}\text { - 'Just being with the group.' [Participant A] } \\
\text { - 'I wasn't well today (I have a headache) but I just wanted to come and listen....' }[\text { Participant } C] \\
\text { - 'Everything about being in a group with everyone.' [Participant } E] \\
\text { - 'Group process - hearing everyone else and that people have the same problems as me.' [Participant F] } \\
\text { - 'Realizing where I am in relation to something another group member said that I could identify with - a } \\
\text { recent experience took me away from humanity. The compassion exercise made me reconnect to } \\
\text { humanity - compassion for the other person. But **** (group member name) can't develop any } \\
\text { compassion for the person that hurt her because she was angry. I then thought about me.' [Participant } G] \\
\text { - 'Life compass - especially individual work as this allowed me to talk about my issues in private and then } \\
\text { group share what I wanted to.' [Participant B] } \\
\text { - 'Coming here and sharing problems makes me feel like I'm not the only one who has problems.' } \\
\text { [Participant E] } \\
\text { - I really feel (facilitator name) is an amazing, very helpful psychologist ... I've been very impressed by } \\
\text { (facilitator name). She was genuine, sincere and natural ... Overall making it helpful.' [Participant E] } \\
\text { - 'As you know I suffered with mouth cancer and I went through such a long process of surgery and stages } \\
\text { of recovery... I felt socially shy and suffered from depression and anxiety when I had to communicate } \\
\text { with other people. But today I share and today I got the news that my cancer is in remission (applause in } \\
\text { group).' [Participant } G]\end{array}$ \\
\hline
\end{tabular}


Table 3. Continued

\begin{tabular}{|c|c|c|}
\hline Master theme & Sub-themes & Some indicative quotes from the data \\
\hline \multirow[t]{2}{*}{$\begin{array}{l}\text { 2. Change } \\
\text { Factors }\end{array}$} & $\begin{array}{l}\text { Emotional } \\
\text { transitions }\end{array}$ & $\begin{array}{l}\text { - 'I dedicated my whole life to my son and I only ever thought about him, always him, my life was } \\
\text { consumed by my son and his problems, and how his problems affect the whole family ... But now I feel I } \\
\text { can deal with the stress ... For the past } 15 \text { years I felt I couldn't change my son, I was helpless. I even had } \\
\text { my own therapy throughout this time, but I just couldn't accept his problem. But now I don't feel I need } \\
\text { to change his problems in order to be a good mother. I can still love him. This feels good.' [Participant B] } \\
\text { - ‘. . The compassion exercise actually helped me to realize that I have moved on in my problem ... } \\
\text { Imagining him as a 5-year-old child and giving him compassion was the moment something shifted.' } \\
\text { [Participant } C \text { ] } \\
\text { 'I was always blaming other people ... The compassion exercise helped me to notice the other person's } \\
\text { vulnerability...' [Participant } D]\end{array}$ \\
\hline & $\begin{array}{l}\text { New } \\
\text { perspectives }\end{array}$ & $\begin{array}{l}\text { - 'I found out that there are so many out there who have problems like I do. I know now that I am not the } \\
\text { only one who has difficulties, which means I can go on ...' [Participant A] } \\
\text { - 'All helpful but good to get direction - Life Compass.' [Participant B] } \\
\text { - 'I know how stress was affecting my body - but the sessions helped me realize how my psychological } \\
\text { problems affect my health ...' [Participant C] } \\
\text { - 'Value cards gave me a direction so I could find my way better. Acceptance is the solution. Accepting } \\
\text { difficulties you can't change and finding a way to live with it...' [Participant } C] \\
\text { - 'Good to have information about stress - learning more about stress and how it affects my body was } \\
\text { interesting.' [Participant D] } \\
\text { - 'Video on blind man - it was very encouraging and puts life in perspective. It opened up opportunities } \\
\text { (possibilities) in my mind, I can learn English, I can do what I want because I am healthy physically and } \\
\text { mentally.' [Participant E] } \\
\text {-'The value of Justice in my life came up very strongly in this group. I've been in therapy for a long time } \\
\text { but this group gave me a better understanding of how I am relating to my problem. I always blamed } \\
\text { others and now have developed insight in to how I am creating my suffering - I am learning now to take } \\
\text { responsibility for my role in my life...' [Participant F] } \\
\text { - 'Choices discussion - understanding making bad choices is not a disaster but an opportunity to turn bad } \\
\text { choices into good choices'. [Participant F] }\end{array}$ \\
\hline
\end{tabular}


Table 3. Continued

\begin{tabular}{|c|c|c|}
\hline Master theme & Sub-themes & Some indicative quotes from the data \\
\hline $\begin{array}{l}\text { 2. Change } \\
\text { Factors }\end{array}$ & $\begin{array}{l}\text { Different } \\
\text { actions }\end{array}$ & $\begin{array}{l}\text { - ‘... enjoyment to my life, e.g. I have been lighting candles and preparing food and I enjoy my life again } \\
\text { with my son ...' [Participant } A] \\
\text { - 'Now I am making time for myself ... now I feel I can start to look after myself as well }\{\text { as other family } \\
\text { members }\} \text {. [Participant } B] \\
\text { - 'All of it helpful and I am practising the mindfulness...' [Participant } F]\end{array}$ \\
\hline \multirow[t]{3}{*}{$\begin{array}{l}\text { 3. Reflections/ } \\
\text { Considerations }\end{array}$} & Group feedback & $\begin{array}{l}\text { - 'Physically and socially the group has helped me... Every session has been helpful for me, I got } \\
\text { something from every session.' [Participant B] } \\
\text { - ‘... I also have noticed that all the others enjoyed the last session. If there was a group about compassion, } \\
\text { I've would have attended. I am very glad that I've joined this course...' [Participant G] }\end{array}$ \\
\hline & $\begin{array}{l}\text { Interpreting } \\
\text { language }\end{array}$ & • 'I would like it to be just Turkish rather than having an interpreter in the session.' [Participant E] \\
\hline & Use of metaphor & $\begin{array}{l}\text { - 'Applying the bus metaphor was good to demonstrate as it helped me to apply it to my life situation and } \\
\text { made me more aware of my thoughts and emotions.' [Participant A] } \\
\text { - 'Turkish delight exercise - we have so many blessings in our lives and most of the time don't even notice } \\
\text { - this exercise helped me to remember.' [Participant A] } \\
\text { - 'Bus metaphor helped me to identify which passengers to get rid of and kick off the bus!' [Participant B] } \\
\text { - 'Storytelling - it focused me to look at the story of my life.' [Participant C] } \\
\text { - '... life compass helped to focus me in a practical way'. [Participant D] } \\
\text { - 'Value cards were most important. Made me think of the different areas of my life and what is important } \\
\text { to me. It gave me ideas and also reminded me of what is important...' [Participant E] }\end{array}$ \\
\hline
\end{tabular}




\section{Theme 1: Group Process}

This theme consisted of two sub-themes linked to aspects of attendance in a therapeutic group.

\section{Interpersonal factors}

All participants commented on various interpersonal processes as being some of the most important aspects of the group. The relationships that developed appeared to link with feeling valued, accepted and connected with others. Five participants highlighted that 'sharing and being listened to' were integral within the group. Four participants appreciated hearing the stories and views of others, as it allowed them to reflect on their own experience. The idea of identifying with someone else's story, not feeling alone and learning from others was highlighted by six participants and appeared to be 'powerful' within the group and associated with 'sharing'.

'The most important part of the group for me was the connection. The relationships in the group, sharing our stories, listening to each other ...' [Participant A]

'... the group process of hearing other peoples' opinions and listening to them sharing their problems is important as it helps me to think about what I experience.' [Participant D]

\section{A safe space}

There were also various indicators that the group created a safe space, where people felt able to experience emotions and to fully participate (derived from six participants' comments). This safety seemed to arise from developing relationships between group members, the therapeutic alliance with facilitators and task structure (group sharing and some 1:1 work) where people could experiment with their degree of comfort in sharing personal or difficult material.

'For me also, the group process was very powerful. Being listened to and attended to and I felt a sense of freedom - it felt safe to talk and be accepted ... Gave me a feeling of being valued and I felt appreciation for the group space you created ...' [Participant $F$ ]

'I felt that I was in safe hands. Special thanks to \{facilitator names\}.' [Participant B]

The interpersonal dynamics and group space created an environment where individuals felt connected, validated and comfortable to express themselves and learn from each other. Although this emerged as a positively slanted theme, all members of the group did not hold this view. One participant mentioned that although they found it helpful to talk about their 'problems', at times they worried about the group not being 'confidential or safe enough' in relation to fears of exposure within the community setting. However, overall it did suggest that this is a generally accessible intervention.

\section{Theme 2: Change Factors}

This theme consisted of three sub-themes linked to changes within emotion, thoughts and behaviour.

\section{Emotional transitions}

Five participants identified accessing emotion as a key feature of the group. The group facilitated the discovery of more positive emotions, which participants found liberating, either 
in the actual experience itself or in knowing that this emotion can exist within them. Some of the most frequent emotions identified by all participants were 'compassion, kindness and enjoyment/happiness':

'I realized I was carrying a lot of anger too but not any more as I was able to access human kindness today and this made me aware of where I am.' [Participant $G]$

'... it was a releasing feeling and it was so good to notice that I don't live with hatred anymore and

I actually have compassion and kindness in me. The experience I had with this person made me feel like I wasn't human anymore - I felt like a monster - but the compassion exercise helped me to reconnect with my humanity and this was powerful and healing ...' [Participant $C$ ]

Six participants commented on accessing new emotions towards others in their lives, with five participants linking this to people whom they had difficult feelings towards. This enabled participants to notice other aspects of their experience, and relate to these individuals or memories in a different way. This appeared to assist participants to believe that they could move forward from the confines of their difficulties:

'I still feel the feeling of compassion for this person since the last session - it was such a strong feeling and I went through the most powerful emotional process - I had to leave the session. It shifted something ...' [Participant $F]$

Four participants also indicated that they felt more able to relate differently to some emotions, suggesting acceptance of difficult feelings. These participants identified a relationship between accessing new or different emotions (e.g. compassion) and deepening their capacity to experience other difficult feelings. The mindfulness and compassion exercises stood out as useful to all participants, as a facilitator to 'being' with feelings, as well as being with their thoughts and fostering new ideas associated with the 'problems' themselves.

'... problems I tried to change it to thinking about positive things. But that didn't work either because I still felt angry. Digging in to my problems doesn't help. But the mindfulness practice helped me to be able to be with my problem, and relate to it in a different way ...' [Participant $C$ ] '... I used to have so much anger and hatred I could not feel or connect with compassion but now I am learning to be with my anger and live with it and also feel compassion.' [Participant $F$ ]

\section{New perspectives}

All participants' responses highlighted new understandings of their difficulties, which were often summaries of the journey of their 'problems' and their relationship to them. A new 'awareness' seemed to emerge involving patterns of functioning in the moment, what maintained difficulties and how problems impeded individuals from moving forward in life:

'I dedicated my whole life to my son and I only ever thought about him, always him, my life was consumed by my son and his problems ... Before, I couldn't concentrate when talking to friends; I was always distracted by my thoughts and worries for my son. I didn't go out or have any social contact for 2 years ...' [Participant $B$ ]

All participants reflected that the group enabled them to look 'at things from a different perspective'. This facilitated shifts to a narrative that was more positive, hopeful or understanding and accepting of their current situation and the future. The view of not being 
alone in experiencing difficulties (identified by six participants) appeared to be powerful amongst the group and this collective experience seemed to provide participants with a sense of strength:

'Feeling that I am not the only one suffering with my problems is most important thing for me... The group is helping me feel that I am not alone with my problems.' [Participant $C$ ]

Five participants also identified a new acceptance of difficulties or past events. There appeared to be a letting go of a fight to change others or themselves, to being able to live with the experience of distress or the problem and still engage with things they valued. There was an evident change in five participants' sense of personal agency, seeing themselves as more able to 'deal with' the difficulties they faced in life. These 'new understandings' appeared to foster a feeling of empowerment and increase emotional resilience, which helped to move participants into a position from which they could become unstuck, view other possibilities and experience value in life again.

'... but thanks to these sessions I feel I can deal with my problems - I can live with my problems

- and still have some quality of enjoyment to my life... The group gave me something: the realization that I can have problems but still enjoy my life'. [Participant A]

Overall, participants' responses clearly outlined various types of new or reinvigorated perspectives and emotions, which were nurtured from participating in the group. All participants identified various group tasks acting as catalysts (e.g. life compass, value cards exercise), by providing some 'direction, focus and clarity', in their reflections and identification of their difficulties, values, new understandings and connection with emotions. In addition, four participants commented on experiencing particular shift moments within the group that illuminated these new views or emotions:

'...I had a moment of eureka, realizing that even when I don't do anything, and don't make a decision - that's still making a choice.' [Participant $B$ ]

These 'shift' moments were described as an experience that created the foundation for new possibilities of 'living life' despite experiencing difficulties.

\section{Different actions}

When reflecting on the experience of the group, six participants started to reconnect with previous values, value-based activities or started something new (i.e. an activity, 'making time for things' or strategies to cope with experiences):

'Group helped me reconnect with the good things in life that I used to do before I became ill such as buying flowers and exercising more...' [Participant $E]$

The group appeared to encourage participants to actively do something different associated with their values, interests and enjoyment, or managing difficulties. These activities also linked with new perspectives about being 'able to deal with stress' and other difficulties in life, which included having some time to attend to one's own needs. 


\section{Theme 3: Reflections/Considerations}

\section{Group feedback}

All participants often reported a favourable emotion towards attending the group, towards specific sessions or tasks and the environment the group created:

'... I'm very happy to be here.' [Participant $A]$

The feedback from all participants suggests that the ACT group was seen as helpful, with four participants interested in more sessions or attending future groups of this kind. All participants expressed that 'all of it' or 'bits of everything' were useful and there was a suggestion (from five participants) that each session provided something additional to further their understanding. There was a clear interest in building on concepts and further work around emotions, with four participants highlighting compassion and anger. The group seemed to be an enjoyable experience, with participants appreciating the group process, indicating this format as an acceptable intervention.

\section{Interpreting language}

Two participants reflected on the difficulties of interpretation for the group. This was associated with preference, a disrupted connection to the group material and other participants, and an inhibited experience of emotional safety when sharing personal events:

'I did not like the fact there were two languages. Perhaps something was lost in translation or I did not feel connected to the group, there was no flow in conversation, for example I was about to share something sensitive and felt like I was interrupted as it had to be translated ...' [Participant $D]$

This was a significant comment, given the opposing views of the majority of participants who indicated strong attachments and confidence in sharing 'sensitive' material within the group. It highlights the complex process of translating and the possible difficulties in cultivating a group alliance within this context. Although the majority of participants did not comment negatively on the effect of translating, it is a valuable consideration given the process of translating in a group setting with multiple people and perspectives.

\section{Use of metaphor}

Six participants shared how the use of tasks, involving practical exercises, cultural stories, metaphors and hearing other peoples' experiences and stories were important in being able to think about their situation in a manageable way:

'I slowly understood how the value cards related to the life compass - how they connected. And that was the most important thing for me.' [Participant $A$ ]

'The farmer and horse story - there is a necklace in Turkey with words inscribed: good things bring sadness afterwards and bad things bring good things afterwards.' [Participant E]

This suggests that the use of particular tasks and concepts within ACT was accessible and aided a connection to cultural narratives and new ideas. 


\section{Discussion}

This pilot project set out to explore the cultural flexibility of the ACT model for Turkishspeaking communities living in an inner London borough. There are no consistent guidelines in the literature for modifying interventions using cultural adaptation frameworks (Rathod, 2017). Thus the acceptability of the adapted 'Acceptance with Mindfulness and Compassion' ACT group was based on the extent to which it took into account participants' social world, cultural values, context and reality, its ability to respond to the therapeutic needs of participants, and cultural relevance, as indicated by patient-reported outcome data. Due to the pilot status of this explorative study, only tentative conclusions can be drawn.

The results demonstrated an overall positive effect of the culturally adapted ACT intervention. Participants showed significant improvements on measures of depression, anxiety and psychological distress. In terms of qualitative data, the thematic analysis suggested that the group was experienced as enjoyable, useful, and would be an accessible and acceptable format for ongoing therapeutic work for Turkish-speaking communities. Although pilot in nature, these results are consistent with the growing body of research suggesting the efficacy of culturally adapted ACT interventions in both client improvements and the acceptability and accessibility across cultures (Bernal et al., 2009; Griner and Smith, 2006; Stewart et al., 2016; White and Ebert; 2014; Woidneck et al., 2012).

This study reports large effect sizes for the group, which is in line with other studies which indicate that ACT interventions yield between a small-to-large effect size varying by study design, across psychological difficulties and for client groups from 'non-dominant cultural and/or marginalized backgrounds' (Forman et al., 2007; Fuchs et al., 2013; Johns et al., 2016; Powers et al., 2009; Vøllestad et al., 2012). However, although Hedges' correction was used to calculate a more conservative effect size due to a small sample size, these Hedges' measurements (gav or grm) are not completely unbiased in calculating effect size (Cumming, 2012; Lakens, 2013) and wide confidence intervals mean that the findings are interpreted tentatively.

At an individual level, the improvements observed were reliable (between 71.4 and 85.7\%), although fewer participants experienced both a reliable and clinically significant reduction in symptomology (between 28.6 and $42.8 \%$ ). This indicates that the intervention is promising but may need further modification, as there is not a strong enough indication that it leads to clinically significant symptom reduction (scoring below clinical cut-offs for a diagnostic difficulty). However, it is an encouraging start, especially given participants' reliable symptom reduction within the clinical range and the potential effects this may have on quality of life. Further analysis is required with a larger sample and control group, particularly as null findings should be interpreted carefully in pilot studies due to small sample sizes and regarded as inconclusive as opposed to evidence of an absence of effect (Altman and Bland, 1995; Thabane et al., 2010).

From the qualitative analysis, the important themes identified involved noteworthy emotional experiences and changes in one's perspectives on the understanding of their difficulties, the presence of emotions and on future possibilities. Some of these areas appear to reflect ACT aims of changing an individual's relationship to unwanted internal experiences rather than changing the experience itself and promoting psychological flexibility around 'stuck' places (Hayes et al., 2012). This appeared to empower individuals in the group to make different choices despite experiencing hardship and identify valued action to engage in 
within this new view. Secondly, being part of the group appeared to be very powerful (e.g. hearing, connecting and learning from other people's stories), with the significance of these interpersonal processes potentially both in line with the values of collectivist cultures and being facilitators of change (Yalom, 1975). The results suggest that key ACT practices such as mindfulness and acceptance potentially encouraged participants to be more aware, nonjudgmental and willing to reconnect to the parts of their lives that matter to them (i.e. values such as perseverance, compassion, forgiveness, belonging and interconnectedness) which also promoted recovery. Furthermore, the group context supported participants to connect with resources in the face of adversity, step towards psychological flexibility and learn more effective ways of responding to distressful internal experiences. These findings support the potential usefulness of the ACT model with Turkish-speaking communities, as well as the supportive function of being part of a group (e.g. not feeling alone) and compassion eliciting exercises (that are related - but not integral to - an ACT model).

\section{Limitations}

This is a pilot study and so the robustness of the findings is limited by a number of factors, including a small sample size and lack of control group, which means it cannot fully ascertain whether the ACT interventions were key to the improvements found. However, given the context of the pilot, it provides an opportunity to inspire the provision of culturally competent care in clinical practice. It also warrants larger standardized research utilizing control groups and implementing follow-up measures to determine whether the small improvements found in this study would be maintained and to help to further understand the potential benefits of acceptance-based interventions for Turkish-speaking communities in the UK.

Furthermore, there may have been other effects not measured within the study, e.g. compassion and acceptance, which may be of consideration given that these areas were reflected in the themes identified and within the ACT approach. The measures used in the current study were not ACT specific, but based on psychopathology, which was a pragmatic service related decision based on the requirement to be compliant with the IAPT Framework for assessing outcomes (Gyani et al., 2013). Future research could include a range of different ACT appropriate measures that would enable us to detect effects in specific ACT domains. Analysis using these measures may also be able to assess potential mechanisms of change, proposed by ACT interventions more widely.

Notwithstanding the above limitations, the findings from this pilot study suggest some value in tailoring ACT to the cultural needs of Turkish-speaking communities in the UK. Given the context of the BME Access Service, it is useful to assess and report on interventions implemented to share and inspire potential clinical practice relating to the accessibility and equity of service provision across diverse populations. Overall, the current study underlines the importance of community consultation and partnership working in the development of culturally responsive and accessible therapeutic interventions for BME communities in East London.

\section{Main points}

Based on these preliminary findings, the partnership project has made successful steps in meeting its stated aims of: 
- Developing a culturally relevant adaptation of Livheim's (2014) ACT group therapy protocol.

- Demonstrating that the ACT model may be amenable to successful adaptation across different cultural settings. This finding supports the clinical utility of ACT as a potentially effective, culturally acceptable therapeutic approach for Turkish-speaking communities living in an urban UK setting in supporting psychological well-being.

- Promoting community collaboration and the need to provide culturally relevant and accessible psychotherapies for ethnically diverse populations.

Given the current climate of funding constraints and a drive to develop models of best practice, there is an ever-increasing need to carry out further evaluative research that explores culturally adapted psychological interventions, contextually tailored to the specific needs of different BME communities. The clinical usefulness of the current pilot study may serve to support future partnerships in developing creative and innovative adaptations appropriate to different cultural contexts, as well as encouraging larger research initiatives. This may further improve the cultural competency of service provision, leading to more equitable access to talking therapies, enhanced service user experience and better recovery outcomes for BME communities across both statutory and voluntary sector organizations in the UK.

\section{Acknowledgements}

The authors wish to acknowledge Derman as the partnership organization for their contribution to this project and assistance with community consultation, recruitment and delivery of the group, and Feyza Özcan Balaban for additional language support. Thanks are due to Dr Shanaya Rathod for helpful comments on an earlier draft of the manuscript. Finally, thanks to all the participants for their involvement.

\section{Ethical statement}

The authors have abided by the Ethical Principles of Psychologists and Code of Conduct as set out by the APA. This project constituted service-related research that evaluated an aspect of service provision not requiring ethical approval.

\section{Conflicts of interest}

Aradhana Perry, Chelsea Gardener, Joseph E. Oliver, Çiğdem Taş and Cansu Özenç have no conflicts of interest with respect to this publication.

\section{Financial support}

This project was supported by the City and Hackney Clinical Commissioning Group Psychological Therapies Alliance in East London.

\section{Recommended follow-up reading}

Bhui K, Aslam RW, Palinski A, McCabe R, Johnson MR, Weich S, Singh SP, Knapp M, Ardino V, Szczepura A (2015). Interventions designed to improve therapeutic communications between black 
and minority ethnic people and professionals working in psychiatric services: a systematic review of the evidence for their effectiveness, Health Technology Assessment 19, 1-173.

Fuchs C, Lee JK, Roemer L, Orsillo SM (2013). Using mindfulness-and acceptance-based treatments with clients from non-dominant cultural and/or marginalized backgrounds: clinical considerations, meta-analysis findings; and Introduction to the special series: clinical considerations in using acceptance-and mindfulness-based treatments with diverse populations. Cognitive and Behavioural Practice 20, 1-12.

Hayes SC, Strosahl K, Wilson KG (1999). Acceptance and Commitment Therapy: An Experiential Approach to Behavior Change. New York: Guilford Press.

Rathod S, Kingdon D, Pinninti N, Turkington D, Phiri P (2015). Cultural Adaptation of CBT for Serious Mental Illness: A Guide for Training and Practice. Wiley-Blackwell.

\section{References}

Altman DG, Bland JM (1995). Absence of evidence is not evidence of absence. British Medical Journal 311, 485.

A-Tjak JG, Davis ML, Morina N, Powers MB, Smits JA, Emmelkamp PM (2015). A meta-analysis of the efficacy of acceptance and commitment therapy for clinically relevant mental and physical health problems. Psychotherapy and Psychosomatics 84, 30-36.

Barkham M, Bewick B, Mullin T, Gilbody S, Connell J, Cahill J et al. (2013). The CORE-10: a short measure of psychological distress for routine use in the psychological therapies. Counselling and Psychotherapy Research 13, 3-13.

Bernal G, Bonilla J, Bellido C (1995). Ecological validity and cultural sensitivity for outcome research: issues for the cultural adaptation and development of psychosocial treatments with Hispanics. Journal of Abnormal Child Psychology 23, 67-82.

Bernal G, Jiménez-Chafey M, Domenech Rodríguez M (2009). Cultural adaptation of treatments: a resource for considering culture in evidence-based practice. Professional Psychology: Research and Practice 40, 361-368.

Berzon B, Pious C, Farson RE (1963). The therapeutic event in group psychotherapy: a study of subjective reports by group members. Journal of Individual Psychology 19, 204-212.

Bhui K, Aslam RW, Palinski A, McCabe R, Johnson MR, Weich S, Singh SP, Knapp M, Ardino V, Szczepura A (2015). Interventions designed to improve therapeutic communications between black and minority ethnic people and professionals working in psychiatric services: a systematic review of the evidence for their effectiveness, Health Technology Assessment 19, 1-173.

Bhui K, Bhugra D, McKenzie K (2000). Maudsley discussion paper no. 8: Specialist services for minority ethnic groups. Available at: https://www.kcl.ac.uk/ioppn/contact/mentalhealth/ publications/discussion-papers/assets/mdp08.pdf (accessed 10 February 2018).

Bhui K, Olajide D (eds) (1999). Mental Health Service Provision for a Multi-Cultural Society. London: WB Saunders.

Bloch S, Reibstein J, Crouch E, Holroyd P, Themen J (1979). A method for the study of therapeutic factors in group psychotherapy. British Journal of Psychiatry 134, 257-263.

Borenstein M, Hedges LV, Higgins JPT, Rothstein HR (2009). Introduction to Meta-Analysis. West Sussex: Wiley.

Braun V, Clarke V (2006). Using thematic analysis in psychology. Qualitative Research in Psychology 3, 77-101.

City and Hackney Clinical Commissioning Group (2015). Mental Health Programme Board minutes. Available at: http://www.cityandhackneyccg.nhs.uk/Downloads/About\%20Us/Programme\% 20Boards/Mental\%20Health/March\%202015\%20Meeting\%20Pack.pdf 
Cumming G (2012). Understanding the New Statistics: Effect Sizes, Confidence Intervals and MetaAnalysis. New York, NY: Routledge.

Department of Health (2009). Improving Access to Psychological Therapies (IAPT). Available at: http://www.iapt.nhs.uk/silo/files/black-and-minority-ethnic-bme-positive-practice-guide.pdf

Enneli P, Modood T, Bradley H (2005). Young Turks and Kurds: a set of 'invisible' disadvantaged groups. York, UK: Joseph Rowntree Foundation.

Eylem O, van Bergen DD, Rathod S, van Straten A, Bhui K, Kerkhof AJFM (2016). Canına kıymak - 'crushing life energy': a qualitative study on lay and professional understandings of suicide and help-seeking among Turkish migrants in the UK and in the Netherlands. Available at: https://research.vu.nl/en/publications/can\%C4\%B1 na-k\%C4\%B1ymak-crushing-life-energy-aqualitative-study-on-lay-and

Forman EM, Herbert JD, Moitra E, Yeomans PD, Geller PA (2007). A randomized controlled effectiveness trial of acceptance and commitment therapy and cognitive therapy for anxiety and depression. Behavior Modification 31, 772-799.

Fountain J, Patel K, Buffin J (2007). Community Engagement: the Centre for Ethnicity and Health Model. Foundation Regenboog AMOC.

Fuchs C, Lee JK, Roemer L, Orsillo SM (2013). Using mindfulness-and acceptance-based treatments with clients from non-dominant cultural and/or marginalized backgrounds: clinical considerations, meta-analysis findings; and Introduction to the special series: clinical considerations in using acceptance- and mindfulness-based treatments with diverse populations. Cognitive and Behavioural Practice 20, 1-12.

Furnham A, Malik R (1994) Cross cultural beliefs about depression. International Journal of Social Psychiatry 40, 106-123.

Griner D, Smith T (2006) Culturally adapted mental health intervention: a meta-analytic review. Psychotherapy: Theory, Research, Practice, Training 43, 531-548.

Gyani A, Shafran R, Layard R, Clark DM (2013). Enhancing recovery rates: lessons from year one of IAPT. Behaviour Research and Therapy 51, 597-606.

Hall GCN, Hong JJ, Zane NWS, Oanh LM (2011) Culturally competent treatments for Asian Americans: the relevance of mindfulness and acceptance-based psychotherapies. Clinical Psychology 18, 215-231.

Hayes SC, Luoma JB, Bond FW, Masuda A, Lillis J (2006). Acceptance and commitment therapy: model, processes and outcomes. Behaviour Research and Therapy 44, 1-25.

Hayes SC, Strosahl KD, Wilson KG (2012). Acceptance and Commitment Therapy: The Process and Practice of Mindful Change. New York: Guilford Press.

Hoseini SM, Rezaei AM, Azadim MM (2014) Effectiveness of acceptance and commitment group therapy of the self-management of type 2 diabetes patients. Journal of Clinical Psychology 5, 55-64.

Jacobson NS, Truax P (1991). Clinical significance: a statistical approach to defining meaningful change in psychotherapy research. Journal of Consulting and Clinical Psychology 59, 1219.

Johns LC, Oliver JE, Khondoker M, Byrne M, Jolley S, Wykes T, Joseph C, Butler L, Craig T, Morris EM (2016). The feasibility and acceptability of a brief Acceptance and Commitment Therapy (ACT) group intervention for people with psychosis: the 'ACT for life' study. Journal of Behavior Therapy and Experimental Psychiatry 50, 257-263.

Kroenke K, Spitzer RL (2002). The PHQ-9: a new depression diagnostic and severity measure. Psychiatric Annals 32, 509-515.

Kuzulugil SS (2010). A classification Turkish cultural values. Paper presented at the International Conference on Social Sciences, ICSS.

La Roche M, Lustig K (2013). Being mindful about the assessment of culture: a cultural analysis of culturally adapted acceptance-based behavior approaches. Cognitive and Behavioral Practice 20, $60-63$. 
Lakens D (2013). Calculating and reporting effect sizes to facilitate cumulative science: a practical primer for t-tests and ANOVAs. Frontiers in Psychology 4, 863.

Lieberman M, Yalom I, Miles M (1973). Encounter Groups: First Facts. New York: Basic Books.

Livheim F (2014). 'ACT - promoting mental health and resilience' Group. Life Compass. ACT Organisation.

Lundgren T, Dahl J, Melin L, Keis B (2006). Evaluation of acceptance and commitment therapy for drug refactory epilepsy: a randomized controlled trial in South Africa. Epilepsia 47, 21732179.

Lundgren T, Dahl J, Yardi N, Melin J (2008) Acceptance and Commitment Therapy and yoga for drug-refractory epilepsy: a randomized controlled trial. Epilepsy and Behavior 13, 102-108.

Masuda A (2014). Psychotherapy in cultural context: an overview. In A Masuda (ed), Mindfulness and Acceptance in Multicultural Competency. Context Press.

Mirdal GM (2012). Mevlana Jalāl-ad-Dīn Rumi and Mindfulness. Journal of Religion and Health, 51, 1202-1215.

Powers MB, Zum Vörde Sive Vörding MB, Emmelkamp PM (2009). Acceptance and commitment therapy: a meta-analytic review. Psychotherapy and Psychosomatics 78, 73-80.

Rathod S (2017). Contemporary psychotherapy and cultural adaptations. Journal of Contemporary Psychotherapy 47, 61-63.

Rathod S, Phiri P, Harris S, Underwood C, Thagadur M, Padmanabi U, Kingdon D (2013). Cognitive behaviour therapy for psychosis can be adapted for minority ethnic groups: a randomised controlled trial. Schizophrenia Research 143, 319-326.

Ruiz FJ (2010). A review of Acceptance and Commitment Therapy (ACT) empirical evidence: correlational, experimental psychopathology, component and outcome studies. International Journal of Psychology and Psychological Therapy 10, 125-162.

Spitzer RL, Kroenke K, Williams JB, Lowe B (2006). A brief measure for assessing generalized anxiety disorder: the GAD-7. Archives of Internal Medicine 166, 1092-1097.

Stewart C, White R, Ebert B, Mays I, Nardozzi J, Bockarie H (2016). A preliminary evaluation of acceptance and commitment therapy (ACT) training in Sierra Leone. Journal of Contextual Behavioral Science 5, 16-22.

Tas N, Guden M, Tekin H, Guler I, Doner F, Kalen G (2008) Voice of men: mental health needs assessment of Turkish, Kurdish and Cypriot men in Hackney. Community Engagement Project, NIMHE Mental Health Programme.

Tseng W-S (2004). Culture and psychotherapy: Asian perspectives. Journal of Mental Health 13, 151161.

Thabane L, Ma J, Chu R, Cheng J, Ismaila A, Rios LP et al. (2010). A tutorial on pilot studies: the what, why and how. BMC Medical Research Methodology 10, 1.

Vøllestad J, Nielsen MB, Nielsen GH (2012). Mindfulness-and acceptance-based interventions for anxiety disorders: a systematic review and meta-analysis. British Journal of Clinical Psychology 51, 239-260.

White R, Ebert B (2014). Working globally, thinking locally: providing psychosocial intervention training in Sierra Leone. Clinical Psychology Forum 258, 41-45.

Woidneck MR, Pratt KM, Gundy JM, Nelson CR, Twohig MP (2012). Exploring cultural competence in acceptance and commitment therapy outcomes. Professional Psychology: Research and Practice 43, 227-233.

Yalom ID (1975). The Theory and Practice of Group Psychotherapy, 2nd edn. New York: Basic Books. 


\section{Learning objectives}

(1) To explore the cultural applicability of the Acceptance and Commitment Therapy (ACT) model to psychological distress in non-western Turkish-speaking communities.

(2) To highlight the importance of community consultation and partnership working with third-sector organizations in the development of culturally relevant and accessible therapeutic interventions. 